\title{
Reaction of $o$-(oxiranylmethyl)benzonitriles with sodium borohydride or Grignard reagent/CuI: a new synthesis of substituted 3-alkyl-3,4-dihydroisocoumarins
}

\author{
Po-Yuan Chen, ${ }^{a}$ You-Feng Wang, ${ }^{a}$ Liang-Yeu Chen,,${ }^{b}$ Guan-Lun Wang, ${ }^{a}$ \\ and Eng-Chi Wang *a \\ ${ }^{a}$ Department of Medicinal and Applied Chemistry, Kaohsiung Medical University, \\ Kaohsiung 807, Taiwan \\ ${ }^{b}$ Department of Medical Research, I-Shou University, Kaohsiung 82445, Taiwan \\ E-mail: enchwa@kmu.edu.tw
}

\begin{abstract}
A new method for the synthesis of substituted 3-alkyl-3,4-dihydroisocoumarins is described. $o$-(Oxiranylmethyl)benzonitriles, prepared from isovanillin in five steps, when reacted with nucleophiles such as sodium borohydride or phenylmagnesium chloride/CuI, undergo an intramolecular cyclization to yield the target compounds in good yields, in one pot.
\end{abstract}

Keywords: Isovanillin, oxiranes, benzonitriles, intramolecular cyclization, 3,4-dihydroisocoumarins

\section{Introduction}

3,4-Dihydroisocoumarins (DHIC), otherwise named 3,4-dihydroisochromen-1-ones, are abundantly distributed in a wide range of natural sources. For examples, DHIC isolated from Kigelia pinnata, ${ }^{1}$ Hydrangea macrophylla, ${ }^{2}$ Cape aloe, ${ }^{3}$ Montrouziera sphaeroidea, ${ }^{4}$ Aloe hildebrandtii, ${ }^{5}$ Cassia siamea, ${ }^{6}$ Caryocar glabrum, ${ }^{7}$ as well as others have been reported. Furthermore, certain DHIC from natural sources have broadly biological activities. DHIC such as isolated from Xyris pterygoblephara exhibiting antifungi activity, ${ }^{8}$ from Aloe vera exhibiting binding activity with human serum albumin, ${ }^{9}$ from Fusarium verticillioides exhibiting antimalarial, antitubercular and antifungal activities, ${ }^{10}$ as well as others. On the other hand, DHIC also play an important core structure for many biologically active compounds. For instance, AI-77-B, a naturally-occurring DHIC which chemically belongs to the amicoumacin family, was isolated from different Bacillus genera exhibiting an antiulcerogenic activity without common side effects. ${ }^{11}$ Because of diverse biological activities, a number of synthetic strategies for DHIC have been developed. The major methods reported include the use of the HeckMatsuda reaction, ${ }^{12}$ radical cyclization mediated by titanocene(III) chloride, ${ }^{13}$ cyclization of $\alpha$ - 
lithiated 2-toluenecarboxylates, ${ }^{14}$ coupling of vinylic halides or triflates with $o$-(1-alkenyl)benzoic acids, ${ }^{15}$ the successive lateral and ortho-lithiations of 4,4-dimethyl-2-(o-toyl)oxazoline, ${ }^{16}$ as well as others. However, those reported methods have some disadvantages including tedious reaction conditions, inaccessible starting materials or reagents, and low yields. Therefore, the development of a mild and efficient method for DHIC is requisite and of interest. On the other hand, the ring-opening of epoxides by various nucleophiles to yield diverse organic compounds has been well documented in organic synthesis. ${ }^{17}$ The addition of various nucleophiles to cyano groups has also been well described. ${ }^{18}$ However, studies on the addition of various nucleophiles to aryl compounds with an adjacent epoxy and cyano substituents has seldom been examined. In our previous study, we reported the reaction of $o$-(oxiranylmethyl)benzonitrile intermediates with $\mathrm{TBAB} / \mathrm{NaCN}$ to yield various substituted 3,4-dihydroisoquinolin-1-ones. ${ }^{19}$ Continuing our work on benzoheterocycles, ${ }^{20}$ we herein report the synthesis of substituted 3-alkyl-3,4dihydroisocoumarins from the reaction of $o$-(oxiranylmethyl)benzonitriles with nucleophiles such as sodium borohydride and Grignard reagent in the presence of copper iodide (Scheme 1).

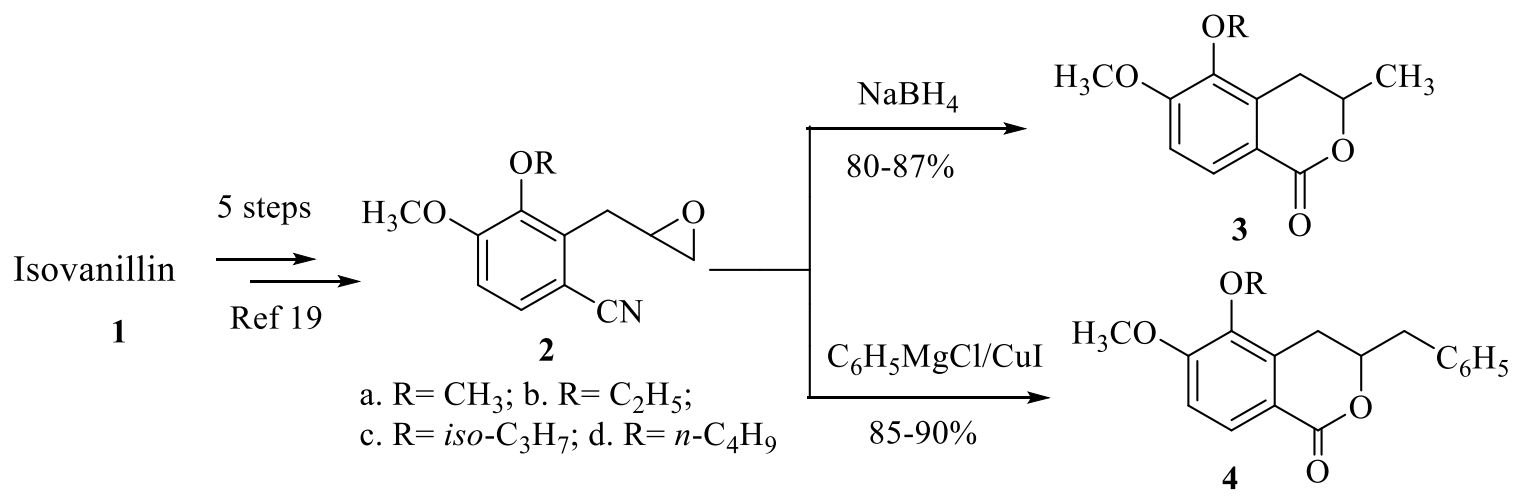

Scheme 1. Synthesis of 3-alkyl-3,4-dihydroisocoumarins from $o$-(oxiranylmethyl)benzonitriles with $\mathrm{NaBH}_{4}$ and $\mathrm{C}_{6} \mathrm{H}_{5} \mathrm{MgCl} / \mathrm{CuI}$ nucleophiles.

\section{Results and Discussion}

In order to optimize the reaction conditions, compound $\mathbf{2 a}$ used as a model reaction was allowed to react with $\mathrm{NaBH}_{4}$ under various conditions. The given results showed that 5,6-dimethoxy-3methyldihydroisochroman (3a) together with 1-(2,3-dimethoxy-6-cyanophenyl)-2-propanol (5a) were formed in varying ratios. Compound 3a was produced through a domino sequence, involving ring-opening of the epoxide, followed by the intramolecular cyclization of the forming alkoxide anion with the cyano functional group, and then hydrolysis. Compound 5a was formed by simple ring opening of the epoxide by $\mathrm{NaBH}_{4}$. The results of this model reaction are compiled in Table 1. 
Table 1. The reaction of compound 2a with $\mathrm{NaBH}_{4}$ under various conditions to yield 3a and 5a

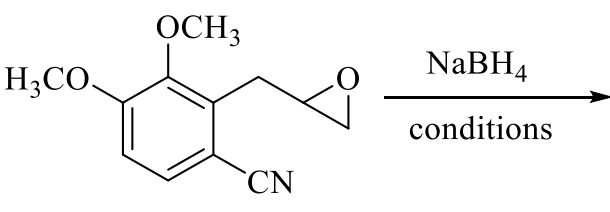

$\mathbf{2 a}$<smiles>COc1ccc2c(c1OC)CC(C)OC2=O</smiles>

$3 \mathbf{a}$<smiles>COc1ccc(C#N)c(CC(C)O)c1OC</smiles>

$5 \mathbf{a}$

\begin{tabular}{ccclccc}
\hline Entry & $\begin{array}{c}\text { Equiv. } \\
\mathrm{NaBH}_{4}\end{array}$ & Solvent & Temp & Time (hr) & 3a $(\%)^{a}$ & 5a $(\%)^{a}$ \\
\hline 1 & 1 & EtOH & rt & 24 & 53 & 45 \\
2 & 1 & EtOH & reflux & 1 & 59 & $33^{20 a}$ \\
3 & 1.5 & EtOH & reflux & 1 & 62 & 38 \\
$4^{b}$ & 1.5 & EtOH & reflux & 1 & 67 & 33 \\
5 & 1.5 & $\mathrm{MeOH}$ & reflux & 1 & 11 & 40 \\
$6^{b}$ & 1.5 & $\mathrm{MeOH}$ & reflux & 1 & 18 & 42 \\
$7^{b}$ & 3.0 & EtOH & reflux & 1 & 80 & - \\
\hline
\end{tabular}

${ }^{a}$ Determined by isolated yields; ${ }^{b}$ Anhydrous solvent was used.

Based on the results reported in Table 1, we concluded that ethanol (entries 1-4) is a better solvent than methanol (entries 5-6) and anhydrous ethanol (entries 4, 7) is the best solvent for the reaction. Three quivalents of $\mathrm{NaBH}_{4}$ (entry 7) is better than 1 or 1.5 equivalents of $\mathrm{NaBH}_{4}$ (entries 1-6), and heating under reflux is better than reaction at room temperature for the production of 3. Thus, the use of excess $\mathrm{NaBH}_{4}$ (3.0 equiv.) in refluxing ethanol (entry 7) gives a high yield (80\%) of 3a from 2a. Based on these conditions, $o$-(oxiranylmethyl)benzonitriles 2a-d gave the target compounds 3a-d in high yields (80-87\%), in the one pot procedure.

All spectral data, such as IR, ${ }^{1} \mathrm{H}-\mathrm{NMR},{ }^{13} \mathrm{C}-\mathrm{NMR}$, EI-MS, and HRMS or EA, are consistent with the 3-methyl-3,4-dihydroisocoumarin structures (3a-d). The IR spectrum of compound 3a, for example, showed absorption at $1707 \mathrm{~cm}^{-1}(\mathrm{C}=\mathrm{O})$ and the ${ }^{1} \mathrm{H}-\mathrm{NMR}$ spectrum exhibited a doublet signal of methyl group bonded to $\mathrm{C}-3(J 6.2 \mathrm{~Hz}$ at $\delta 1.46)$; two double doublet signals at $\delta 2.66$ and 3.13 which respectively have coupling constants $J 16.8,11.4$ and $16.8,3.2 \mathrm{~Hz}$ assigned to $\mathrm{H}-4 \mathrm{a}$ and $\mathrm{H}-4 \mathrm{~b}$; two singlet methoxy signals at $\delta 3.78$ and 3.89 ; a one proton signal at $\delta 4.59$ coupled to neighboring protons assigned as $\mathrm{H}-3$; and two aromatic protons at $\delta 6.88$, and 7.82 with the same coupling constant $J 8.4 \mathrm{~Hz}$ indicating their ortho relationship. In the ${ }^{13} \mathrm{C}$ NMR twelve lines are observed consistent with that required for the structure. The HRMS $(\mathrm{m} / z$ 222.0887) and EA (C, 65.01; H, 6.41) are consistent with the structure. To increase the diversity, $o$-(oxiranylmethyl)benzonitriles (2a-d) were allowed to react with Grignard reagent phenylmagnesium chloride/CuI. At the start of this study, $\mathbf{2 a}$ was reacted with phenylmagnesium 
chloride under various conditions to yield the desired 3-benzyl-5,6-dimethoxydihydroisochroman $\mathbf{4 a}$ and the results are shown in Table 2.

Table 2. The reaction of compound $\mathbf{2 a}$ with $\mathrm{C}_{6} \mathrm{H}_{5} \mathrm{MgBr}$ under various conditions to yield $\mathbf{4 a}$

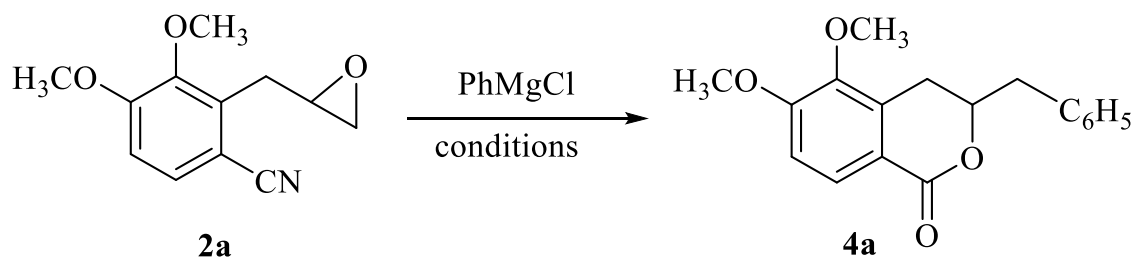

\begin{tabular}{cccccc}
\hline Entry & $\mathrm{Nu}^{-}$ & Additive & Temp & Time (hr) & 4a $(\%)$ \\
\hline 1 & $\mathrm{C}_{6} \mathrm{H}_{5} \mathrm{MgCl}(1.2$ equiv $)$ & - & $0^{\circ}$ - r.t. & 24 & 16 \\
2 & $\mathrm{C}_{6} \mathrm{H}_{5} \mathrm{MgCl}(1.2$ equiv $)$ & $\mathrm{CuI}(0.25$ equiv $)$ & $0^{\text {o-r.t. }}$ & 24 & 47 \\
3 & $\mathrm{C}_{6} \mathrm{H}_{5} \mathrm{MgCl}(1.2$ equiv $)$ & $\mathrm{CuI}(0.50$ equiv $)$ & $0^{\circ}$ - r.t. & 24 & 64 \\
4 & $\mathrm{C}_{6} \mathrm{H}_{5} \mathrm{MgCl}(1.2$ equiv $)$ & $\mathrm{CuI}(1.0$ equiv $)$ & $0^{\circ}$ - r.t. & 24 & 66 \\
5 & $\mathrm{C}_{6} \mathrm{H}_{5} \mathrm{MgCl}(1.2$ equiv $)$ & $\mathrm{CuI}(1.0$ equiv $)$ & reflux & 16 & 11 \\
6 & $\mathrm{C}_{6} \mathrm{H}_{5} \mathrm{MgCl}(2.4$ equiv $)$ & $\mathrm{CuI}(0.5$ equiv $)$ & reflux & 24 & 86 \\
\hline
\end{tabular}

${ }^{a}$ Isolated yield from column chromatography, other by products being neglected.

As shown in Table 2, in the absence of CuI, $\mathbf{4 a}$ was formed in the low yield (16\%) (entry 1) with the exception of entry $5(11 \%)$ which was carried out for a shorter reaction time. This suggests the importance of CuI. A lower amount of Grignard reagent $\mathrm{C}_{6} \mathrm{H}_{5} \mathrm{MgCl}$ (entries 1-5) gave 4a in low to modest yields (11-66\%). On the other hand the reaction of $2 \mathbf{a}$ with excess $\mathrm{C}_{6} \mathrm{H}_{5} \mathrm{MgCl}$ (2.4 equiv)/CuI ( 0.5 equiv) in refluxing THF (entry 6) for $24 \mathrm{hr}$ provided the highest yield for $4 \mathbf{a}(86 \%)$. These conditions were employed for the synthesis of 3-benzyl-3,4dihydroisocoumarins 4a-d from $o$-(oxiranylmethyl)benzonitriles $\mathbf{2 a - d}$, in yields of 85-90\%.

The spectral data including IR, ${ }^{1} \mathrm{H}-\mathrm{NMR},{ }^{13} \mathrm{C}-\mathrm{NMR}$, EI-MS, and HRMS or EA are all consistent with those required for the proposed 5-alkoxy-3-benzyl-6-methoxy-3,4dihydroisocoumarin structures $\mathbf{4 a - d}$. The IR spectrum of compound $\mathbf{4 a}$, for example, shows absorption at $1717 \mathrm{~cm}^{-1}(\mathrm{C}=\mathrm{O})$ indicating the presence of carbonyl group. The ${ }^{1} \mathrm{H}-\mathrm{NMR}$ spectrum exhibits two double doublet signals at $\delta 2.75(\mathrm{dd}, J 16.4,11.2 \mathrm{~Hz}, 1 \mathrm{H})$ and $\delta 3.11$ (dd, $J 16.4,3.2 \mathrm{~Hz}, 1 \mathrm{H}$ ) indicating the presence of $\mathrm{H} 4-\mathrm{a}$ and $\mathrm{H} 4-\mathrm{b}$; other two double doublet signals at $\delta 3.04(\mathrm{dd}, J 14.0,6.8 \mathrm{~Hz}, 1 \mathrm{H}, \mathrm{Ha}-9)$ and $\delta 3.21(\mathrm{dd}, J 14.0,6.0 \mathrm{~Hz}, 1 \mathrm{H}, \mathrm{Hb}-9)$ indicating the presence of $\mathrm{H}-9 \mathrm{a}$ and $\mathrm{H}-9 \mathrm{~b}$ (two benzylic protons), two three-protons singlet signals, each at $\delta$ $3.77,3.93$ indicating two $\mathrm{OCH}_{3}$ groups, and one proton multiplet at $\delta 4.69$ indicating $\mathrm{H}-3$; two one-proton doublet aromatic protons at $\delta 6.91$ and 7.88 with ortho-coupling constant $J 8.4 \mathrm{~Hz}$ indicating the presence of $\mathrm{H}-7$ and $\mathrm{H}-8$ and one multiple signals of five protons at $\delta 7.30$ indicating the presence of aromatic protons of benzyl group. In the ${ }^{13} \mathrm{C}-\mathrm{NMR}$ spectrum of 
compound 4a shows sixteen lines which is consistent with carbon numbers required for the structure 4a. Besides, the data of HRMS (m/z 298.1204) and elemental analysis (C, 72.19; H, 6.03), all data are correct and consistent with the data required for compound $\mathbf{4 a}$.

\section{Conclusions}

We have successfully prepared diverse 3-substituted 3,4-dihydroisocoumarins from the reaction of $o$-(oxiranylmethyl)benzonitriles with nucleophiles $\left(\mathrm{NaBH}_{4}\right.$ or Grignard reagent/CuI). This reaction demonstrates that epoxide ring of $o$-(oxiranylmethyl)benzonitrile opened by nucleophile to give alkoxide anion which attacks the neighboring nitrile to effect the intramolecular cyclization, and this is followed by hydrolysis to yield a series of substituted 3-alkyl-3,4dihydroisocoumarins.

\section{Experimental Section}

General. Melting points were measured with Yanaco micro melting-point apparatus. ${ }^{1} \mathrm{H}-\mathrm{NMR}$ and ${ }^{13} \mathrm{C}$-NMR spectra were obtained on a Varian Unity plus 400 Spectrometer. Chemical shifts were measured in parts per million with respect to TMS. IR spectra were run on a Perkin-Elmer spectrometer (System 2000 FT-IR, series No. 35575). Elemental analyses were recorded on a Heraeus CHN-O Rapid analyzer. Mass spectra were recorded on a Chem/HP/middle spectrometer connected to a Hewlett Packard series II model gas-liquid chromatography. HRMS spectra were performed on a JEOL JMS SX/SX 102A instrument. Silica gel (230-400 mesh) for column chromatography and the pre-coated silica gel plates (60 F-254) for TLC were purchased from E. Merck Co. UV light (254 nm) was used to detect spots on TLC plates after development.

\section{General preparation of 5-alkoxy-6-methoxy-3-methyl-3,4-dihydroisocoumarins (3a-d).} 3-Alkoxy-4-methoxy-2-(oxiranylmethyl)benzonitriles (2a-d) $(2.0 \mathrm{mmol})$ dissolved in EtOH (50 $\mathrm{mL})$ was stirred and added $\mathrm{NaBH}_{4}(0.23 \mathrm{~g}, 6.0 \mathrm{mmol})$ in portions. Then, the reaction mixture was heated to the reflux for $1 \mathrm{hr}$ (monitoring by TLC). The given mixture was quenched with $\mathrm{H}_{2} \mathrm{O}(50 \mathrm{~mL})$, and concentrated in vacuo to remove EtOH. The obtained residue was poured into separating funnel and extracted with $\mathrm{CH}_{2} \mathrm{Cl}_{2}(50 \mathrm{~mL} \times 3)$. The extracted solution was combined and washed with brine $(30 \mathrm{~mL} \times 2)$, dried with $\mathrm{MgSO}_{4}$, and filtered in sequence. The resulting residue was purified from silica gel column chromatography (ethyl acetate: $n$-hexane $=1: 5$ ) to give pure 3a-d. Under the same reaction condition but with insufficient amount of $\mathrm{NaBH}_{4}$ ( 1 mmol), for example, $5 \mathbf{a}$ was obtained.

5,6-Dimethoxy-3-methyl-3,4-dihydroisocoumarin (3a). Compound 3a (0.35 g, 80\%) was obtained as colorless crystals, mp 110-111 ${ }^{\circ} \mathrm{C}\left(\right.$ EtOAc $+n$-hexane) (lit. $\left.{ }^{9} \mathrm{mp} \mathrm{127-128}{ }^{\circ} \mathrm{C}\right), \mathrm{R}_{\mathrm{f}}=$ 0.31 (ethyl acetate: $n$-hexane $=1: 5)$; IR (neat) $v_{\max }: 1707 \mathrm{~cm}^{-1}(\mathrm{C}=\mathrm{O}) ;{ }^{1} \mathrm{H}-\mathrm{NMR}\left(\mathrm{CDCl}_{3}, 200\right.$ 
MHz) $\delta 1.46$ (d, J 6.2 Hz, 3H, H-9), 2.66 (dd, J 16.8, 11.4 Hz, 1H, Hb-4), 3.13 (dd, J 16.8, 3.2 $\mathrm{Hz}, 1 \mathrm{H}, \mathrm{Ha}-4), 3.78,3.89$ (each s, $\left.2 \times 3 \mathrm{H}, 2 \times \mathrm{OCH}_{3}\right), 4.59$ (m, 1H, H-3), 6.88 (d, J 8.4 Hz, 1H, $\mathrm{ArH}), 7.82(\mathrm{~d}, J 8.4 \mathrm{~Hz}, 1 \mathrm{H}, \mathrm{ArH}) ;{ }^{13} \mathrm{C}-\mathrm{NMR}\left(\mathrm{CDCl}_{3}, 50 \mathrm{MHz}\right) \delta 20.9,29.0,55.8,60.5,74.6$, 110.7, 117.8, 127.2, 133.0, 144.5, 156.7, 165.3; EIMS $(70 \mathrm{eV}) \mathrm{m} / z$ (rel. intensity, \%) $222\left(\mathrm{M}^{+}\right.$, 72), 179 (16), 178 (38), 163 (18), 151 (10), 150 (100), 135 (17), 91 (13), 79 (9); HRMS Calcd for $\mathrm{C}_{12} \mathrm{H}_{14} \mathrm{O}_{4}$ : 222.0892. Found: 222.0887; Anal. Calcd for $\mathrm{C}_{12} \mathrm{H}_{14} \mathrm{O}_{4}$ : C, 64.85; H, 6.35. Found: C, 65.01; H, 6.41.

Under the same reaction condition but with insufficient amount of $\mathrm{NaBH}_{4}(1 \mathrm{mmol})$, for example, 3a was obtained in 59\% yield as well as by-product 5a was obtained in $33 \%$ yield.

2-(2-Hydroxypropyl)-3,4-dimethoxybenzonitrile (5a) $(0.15 \mathrm{~g}, 33 \%)$ was obtained as a colorless liquid, $\mathrm{R}_{\mathrm{f}}=0.28$ (ethyl acetate: $n$-hexane $=1: 1$ ); $\mathrm{IR}_{\max }$ (neat) $\mathrm{cm}^{-1}: 2217(\mathrm{CN}), 3407$ $(\mathrm{OH}) ;{ }^{1} \mathrm{H}-\mathrm{NMR}\left(\mathrm{CDCl}_{3}, 400 \mathrm{MHz}\right) \delta 1.30$ (d, J $6.4 \mathrm{~Hz}, 3 \mathrm{H}, \mathrm{H}-3$ '), 2.00 (d, J 5.2 Hz, 1H, OH), 3.02(d, J 6.8 Hz, 2H, H-1'), 3.87, 3.93 (each s, $\left.2 \times 3 \mathrm{H}, 2 \times \mathrm{OCH}_{3}\right), 4.12$ (m, 1H, H-2'), 6.86, 7.41 (each d, $J 8.4 \mathrm{~Hz}, 1 \mathrm{H}, \mathrm{H}-5$ and H-6); ${ }^{13} \mathrm{C}-\mathrm{NMR}\left(\mathrm{CDCl}_{3}, 100 \mathrm{MHz}\right) \delta 23.56,38.50,55.92$, $60.75,68.49$, 105.8110 .9 , 129.7, 131.5, 136.5, 150.8, 156.4; EI-MS (70eV) $\mathrm{m} / \mathrm{z}$ (rel. intensity, \%) $221\left(\mathrm{M}^{+}, 6\right), 178$ (10), 177 (62), 163 (12), 162 (100), 134 (14), 131 (15), 106 (10); HRMS $(\mathrm{EI}, \mathrm{m} / z)$ Calcd for $\mathrm{C}_{13} \mathrm{H}_{14} \mathrm{~N}_{2} \mathrm{O}_{3}: 221.1052$. Found: 221.1053 .

5-Ethoxy-6-methoxy-3-methyl-3,4-dihydroisocoumarin (3b). Compound 3b (0.40 g, 87\%) was obtained as colorless liquid, $\mathrm{R}_{\mathrm{f}}=0.39$ (ethyl acetate: $n$-hexane $=1: 5$ ); IR (neat) $v_{\max }: 1713$ $\mathrm{cm}^{-1}(\mathrm{C}=\mathrm{O}) ;{ }^{1} \mathrm{H}-\mathrm{NMR}\left(\mathrm{CDCl}_{3}, 200 \mathrm{MHz}\right) \delta 1.33\left(\mathrm{t}, J 7.0 \mathrm{~Hz}, 3 \mathrm{H}, \mathrm{OCH}_{2} \mathrm{CH}_{3}\right), 1.48(\mathrm{~d}, J 6.2 \mathrm{~Hz}$, 3H, H-9), 2.68 (dd, J 16.8, 11.4 Hz, 1H, Hb-4), 3.16 (dd, J 16.8, 3.2 Hz, 1H, Ha-4), 3.89 (s, 3H, $\left.\mathrm{OCH}_{3}\right), 4.01$ (q, J 7.0 Hz, 2H, OC$\left.{ }_{2} \mathrm{CH}_{3}\right), 4.56(\mathrm{~m}, 1 \mathrm{H}, \mathrm{H}-3), 6.88$ (d, J $\left.8.8 \mathrm{~Hz}, 1 \mathrm{H}, \operatorname{ArH}\right), 7.84$ $(\mathrm{d}, J 8.8 \mathrm{~Hz}, 1 \mathrm{H}, \mathrm{ArH}) ;{ }^{13} \mathrm{C}-\mathrm{NMR}\left(\mathrm{CDCl}_{3}, 50 \mathrm{MHz}\right) \delta 15.6,21.0,29.4,55.8,68.8,74.7,110.7$, 117.9, 127.1, 133.4, 143.6, 156.9, 165.4; EIMS (70eV) $\mathrm{m} / z$ (rel. intensity, \%) $236\left(\mathrm{M}^{+}, 58\right), 208$ (27), 165 (17), 164 (100), 163 (18), 136(32), 135(28); HRMS Calcd for $\mathrm{C}_{13} \mathrm{H}_{16} \mathrm{O}_{4}: 236.1049$. Found: 236.1043.

5-Isopropoxy-6-methoxy-3-methyl-3,4-dihydroisocoumarin (3c). Compound 3c (0.41 g, 83\%) was obtained as colorless crystals, $\mathrm{mp} 83-84{ }^{\circ} \mathrm{C}, \mathrm{R}_{\mathrm{f}}=0.45$ (ethyl acetate: $n$-hexane $=1: 5$ ); IR (neat) $v_{\max }: 1718 \mathrm{~cm}^{-1}(\mathrm{C}=\mathrm{O}) ;{ }^{1} \mathrm{H}-\mathrm{NMR}\left(\mathrm{CDCl}_{3}, 200 \mathrm{MHz}\right) \delta 1.21\left(\mathrm{~d}, J 6.2 \mathrm{~Hz}, 6 \mathrm{H}, \mathrm{OCH}\left(\mathrm{CH}_{3}\right)_{2}\right)$, 1.44 (d, J 6.2 Hz, 3H, H-9), 2.61 (dd, J 16.8, $11.4 \mathrm{~Hz}, 1 \mathrm{H}, \mathrm{Hb}-4), 3.14$ (dd, J 16.8, $3.2 \mathrm{~Hz}, 1 \mathrm{H}$, Ha-4), 3.85 (s, 3H, $\left.\mathrm{OCH}_{3}\right), 4.40$ (hept, $\left.J 6.2 \mathrm{~Hz}, 1 \mathrm{H}, \mathrm{OC} \underline{\mathrm{H}}\left(\mathrm{CH}_{3}\right)_{2}\right), 4.51$ (m, 1H, H-3), 6.85 (d, $J$ $8.8 \mathrm{~Hz}, 1 \mathrm{H}, \mathrm{ArH}), 7.78(\mathrm{~d}, J 8.8 \mathrm{~Hz}, 1 \mathrm{H}, \mathrm{ArH}) ;{ }^{13} \mathrm{C}-\mathrm{NMR}\left(\mathrm{CDCl}_{3}, 50 \mathrm{MHz}\right) \delta 20.9,22.4,29.8$, 55.7, 74.6, 74.8, 110.5, 117.8, 126.7, 133.7, 142.4, 156.9, 165.4; EIMS (70eV) $\mathrm{m} / \mathrm{z}$ (rel. intensity, \%) 250 (M+, 7), 208 (50), 165 (17), 164 (100), 137 (9), 136 (33), 135 (17), 93 (5); HRMS Calcd for $\mathrm{C}_{14} \mathrm{H}_{18} \mathrm{O}_{4}$ : 250.1205. Found: 250.1200; Anal. Calcd for $\mathrm{C}_{14} \mathrm{H}_{18} \mathrm{O}_{4}$ : C, 67.18; H, 7.25. Found: C, 67.19; H, 7.25.

5-Butoxy-6-methoxy-3-methyl-3,4-dihydroisocoumarin (3d). Compound 3d (0.42 g, 81\%) was obtained as colorless liquid, $\mathrm{R}_{\mathrm{f}}=0.46$ (ethyl acetate: $n$-hexane $=1: 5$ ); IR (neat) $v_{\max }: 1715$ $\mathrm{cm}^{-1}(\mathrm{C}=\mathrm{O}) ;{ }^{1} \mathrm{H}-\mathrm{NMR}\left(\mathrm{CDCl}_{3}, 200 \mathrm{MHz}\right) \delta 0.95\left(\mathrm{t}, J 7.4 \mathrm{~Hz}, 3 \mathrm{H}, \mathrm{OCH}_{2} \mathrm{CH}_{2} \mathrm{CH}_{2} \mathrm{C}_{3}\right.$ ), 1.47 (sixt, $J 7.4 \mathrm{~Hz}, 2 \mathrm{H}, \mathrm{OCH}_{2}-\mathrm{CH}_{2} \mathrm{CH}_{2} \mathrm{CH}_{3}$ ), 1.48 (d, J $6.2 \mathrm{~Hz}, 3 \mathrm{H}, \mathrm{H}-9$ ), 1.71 (quint, J $7.4 \mathrm{~Hz}, 2 \mathrm{H}$, 
$\mathrm{OCH}_{2} \underline{\mathrm{C}}_{2} \mathrm{CH}_{2} \mathrm{CH}_{3}$ ), 2.66 (dd, $J$ 16.8, $11.4 \mathrm{~Hz}, 1 \mathrm{H}, \mathrm{Hb}-4$ ), 3.15 (dd, $J$ 16.8, $3.2 \mathrm{~Hz}, 1 \mathrm{H}, \mathrm{Ha}-4$ ), $3.88\left(\mathrm{~s}, 3 \mathrm{H}, \mathrm{OCH}_{3}\right), 3.91\left(\mathrm{t}, J 7.4 \mathrm{~Hz}, 2 \mathrm{H}, \mathrm{OCH}_{2} \mathrm{CH}_{2} \mathrm{CH}_{2} \mathrm{CH}_{3}\right), 4.55(\mathrm{~m}, 1 \mathrm{H}, \mathrm{H}-3), 6.87$ (d, J 8.4 $\mathrm{Hz}, 1 \mathrm{H}, \mathrm{ArH}), 7.83$ (d, J 8.4 Hz, 1H, ArH); ${ }^{13} \mathrm{C}-\mathrm{NMR}\left(\mathrm{CDCl}_{3}, 50 \mathrm{MHz}\right) \delta 13.8,19.1,20.9,29.2$, 32.2, 55.7, 72.9, 74.7, 110.6, 117.7, 127.1, 133.2, 143.7, 156.9, 165.5; EIMS (70eV) m/z (rel. intensity, \%) 264 (M+, 20), 208 (39), 165 (22), 164 (100), 136 (29), 135 (14), 93(5); HRMS Calcd for $\mathrm{C}_{15} \mathrm{H}_{20} \mathrm{O}_{4}: 264.1362$. Found: 264.1356.

\section{General preparation of 3-benzyl-5-alkoxy-6-methoxy-3,4-dihydroisocoumarins (4a-d).}

3-Alkoxy-4-methoxy-2-(oxiranylmethyl)benzonitriles (2a-d) (2.0 mmol) dissolved in THF (20 $\mathrm{mL})$ was stirred and added copper (I) iodide $(0.22 \mathrm{~g}, 1.17 \mathrm{mmol})$ and then added phenylmagnesium chloride $(2.0 \mathrm{M}$ in THF) $(5.6 \mathrm{mmol})$ dropwise at room temperature. The reaction mixture was continually stirred and heated to the reflux for 1 day (monitoring by TLC). Then, the reaction mixture was quenched with saturated $\mathrm{NH}_{4} \mathrm{Cl}$ solution $(30 \mathrm{~mL})$, and concentrated in vacuo to remove THF. The residue was extracted with $\mathrm{CH}_{2} \mathrm{Cl}_{2}(50 \mathrm{~mL} \times 3)$. The extracted solution was combined and washed with brine $(30 \mathrm{~mL} \times 2)$, dried with $\mathrm{MgSO}_{4}$, and filtered. The filtrate was concentrated in vacuo. The resulting residue was purified from silica gel column chromatography (ethyl acetate: $n$-hexane $=1: 8$ ) to give pure $\mathbf{4 a - d}$.

3-Benzyl-5,6-dimethoxy-3,4-dihydroisocoumarin (4a). Compound 4a (0.50 g, 86\%) was obtained as colorless crystals, $\mathrm{mp} 141-142{ }^{\circ} \mathrm{C}, \mathrm{R}_{\mathrm{f}}=0.31$ (ethyl acetate: $n$-hexane $=1$ : 5); IR (neat) $v_{\max } \mathrm{cm}^{-1}: 1717 \mathrm{~cm}^{-1}(\mathrm{C}=\mathrm{O}) ;{ }^{1} \mathrm{H}-\mathrm{NMR}\left(\mathrm{CDCl}_{3}, 400 \mathrm{MHz}\right) \delta 2.75(\mathrm{dd}, J 16.4,11.2 \mathrm{~Hz}, 1 \mathrm{H}$, Ha-4), 3.04 (dd, $J$ 14.0, 6.8 Hz, 1H, Ha-9), 3.11 (dd, J 16.4, 3.2 Hz, 1H, Hb-4), 3.21 (dd, $J$ 14.0, $6.0 \mathrm{~Hz}, 1 \mathrm{H}, \mathrm{Hb}-9), 3.77,3.93$ (each s, $\left.2 \times 3 \mathrm{H}, 2 \times \mathrm{OCH}_{3}\right), 4.69$ (m, 1H, H-3), 6.91 (d, J 8.4 Hz, 1H, ArH), 7.30 (m, 5H, ArH), 7.88 (d, J 8.4 Hz, 1H, ArH); ${ }^{13} \mathrm{C}-\mathrm{NMR}\left(\mathrm{CDCl}_{3}, 100 \mathrm{MHz}\right) \delta 26.5$, 41.2, 55.9, 60.6, 78.8, 110.8, 118.0, 126.9, 127.5, 128.6, 129.6, 132.9, 136.2, 144.7, 156.9, 165.3; EIMS (70eV) m/z (rel. intensity, \%) 298 (M+, 19), 208 (9), 207 (73), 180 (11), 179 (100), 136 (7), 91 (11); HRMS Calcd for $\mathrm{C}_{18} \mathrm{H}_{18} \mathrm{O}_{4}$ : 298.1205. Found: 298.1204; Anal. Calcd for $\mathrm{C}_{18} \mathrm{H}_{18} \mathrm{O}_{4}$ : C, 72.47; H, 6.08. Found: C, 72.19; H, 6.03.

3-Benzyl-5-ethoxy-6-methoxy-3,4-dihydroisocoumarin (4b) Compound $\mathbf{4 b}(0.55 \mathrm{~g}$, $90 \%)$ was obtained as colorless liquid, $\mathrm{R}_{\mathrm{f}}=0.31$ (ethyl acetate: $n$-hexane $=1: 5$ ); IR (neat) $v_{\max }: 1716 \mathrm{~cm}^{-1}$ $(\mathrm{C}=\mathrm{O}) ;{ }^{1} \mathrm{H}-\mathrm{NMR}\left(\mathrm{CDCl}_{3}, 400 \mathrm{MHz}\right) \delta 1.28\left(\mathrm{t}, J 7.2 \mathrm{~Hz}, 3 \mathrm{H}, \mathrm{OCH}_{2} \mathrm{CH}_{3}\right), 2.73(\mathrm{dd}, J 16.8,10.8$ Hz, 1H, Ha-4), 3.03 (dd, J 14.0, 6.8 Hz, 1H, Ha-9), 3.13 (dd, J 16.4, 3.2 Hz, 1H, Hb-4), 3.21 (dd, $J$ 14.0, $6.0 \mathrm{~Hz}, 1 \mathrm{H}, \mathrm{Hb}-9), 3.91\left(\mathrm{~s}, 3 \mathrm{H}, \mathrm{OCH}_{3}\right), 3.98$ (q, $\left.J 7.2 \mathrm{~Hz}, 2 \mathrm{H}, \mathrm{OC}_{2} \mathrm{CH}_{3}\right), 4.65(\mathrm{~m}, 1 \mathrm{H}$, $\mathrm{H}-3), 6.90$ (d, J 8.4 Hz, 1H, ArH), 7.30 (m, 5H, ArH), 7.86 (d, J 8.4 Hz, 1H, ArH); ${ }^{13} \mathrm{C}-\mathrm{NMR}$ $\left(\mathrm{CDCl}_{3}, 100 \mathrm{MHz}\right) \delta 15.5,26.9,41.2,55.8,68.8,78.8,110.7,118.0,126.8,127.3,128.5,129.6$, 133.2, 136.3, 143.7, 157.0, 165.3; EIMS (70eV) m/z (rel. intensity, \%) $312\left(\mathbf{M}^{+}, 29\right), 222$ (13), 221(97), 194 (22), 193(100), 165(21), 107(12), 91(8); HRMS Calcd for $\mathrm{C}_{19} \mathrm{H}_{20} \mathrm{O}_{4}: 312.1362$. Found: 312.1360 .

3-Benzyl-5-isopropoxy-6-methoxy-3,4-dihydroisocoumarin (4c). Compound 4c (0.55 g, 85\%) was obtained as colorless crystals, $\mathrm{mp} 77-78^{\circ} \mathrm{C}, \mathrm{R}_{\mathrm{f}}=0.32$ (ethyl acetate: $n$-hexane $=1: 5$ ); IR (neat) $v_{\max }: 1718 \mathrm{~cm}^{-1}(\mathrm{C}=\mathrm{O})$; ${ }^{1} \mathrm{H}-\mathrm{NMR}\left(\mathrm{CDCl}_{3}, 400 \mathrm{MHz}\right) \delta 1.20\left(\mathrm{~d}, J 6.4 \mathrm{~Hz}, 3 \mathrm{H}, \mathrm{OCH}\left(\mathrm{C}_{3}\right)_{2}\right)$, 2.69 (dd, $J$ 16.8, 11.2 Hz, 1H, Ha-4), 3.02 (dd, $J$ 14.0, 6.8 Hz, 1H, Ha-9), 3.15 (dd, $J$ 16.8, 3.2 
$\mathrm{Hz}, 1 \mathrm{H}, \mathrm{Hb}-4), 3.21$ (dd, J 13.6, $6.0 \mathrm{~Hz}, 1 \mathrm{H}, \mathrm{Hb}-9), 3.90$ (s, 3H, OCH 3 ), 4.37 (hept, $J 6.4 \mathrm{~Hz}, 1 \mathrm{H}$, $\left.\mathrm{OC} \underline{\mathrm{H}}\left(\mathrm{CH}_{3}\right)_{2}\right), 4.66$ (m, 1H, H-3), 6.89 (d, J $\left.8.8 \mathrm{~Hz}, 1 \mathrm{H}, \mathrm{ArH}\right), 7.31$ (m, 5H, ArH), 7.85 (d, J 8.4 $\mathrm{Hz}, 1 \mathrm{H}, \mathrm{ArH}) ;{ }^{13} \mathrm{C}-\mathrm{NMR}\left(\mathrm{CDCl}_{3}, 100 \mathrm{MHz}\right) \delta 22.3,22.5,27.5,41.2,55.8,75.2,78.9,110.6$, 118.0, 126.8, 127.0, 128.5, 129.5, 133.7, 136.3, 142.5, 157.1, 165.5; EIMS (70eV) $\mathrm{m} / \mathrm{z}$ (rel. intensity, \%) $326\left(\mathrm{M}^{+}, 5\right), 250$ (7), 208 (99), 193 (37), 165 (70), 164 (100), 136 (37), 135 (21); HRMS Calcd for $\mathrm{C}_{20} \mathrm{H}_{22} \mathrm{O}_{4}: 326.1518$. Found: 326.1520. Anal. Calcd for $\mathrm{C}_{20} \mathrm{H}_{22} \mathrm{O}_{4}: \mathrm{C}, 73.60 ; \mathrm{H}$, 6.79. Found: C, 73.28; H, 6.78 .

3-Benzyl-5-butoxy-6-methoxy-3,4-dihydroisocoumarin (4d). Compound 4d (0.60 g, 89\%) was obtained as colorless crystals, $\mathrm{mp} 115-116^{\circ} \mathrm{C}, \mathrm{R}_{\mathrm{f}}=0.31$ (ethyl acetate: $n$-hexane = 1: 5); IR (neat) $v_{\text {max }}: 1719 \mathrm{~cm}^{-1}(\mathrm{C}=\mathrm{O}) ;{ }^{1} \mathrm{H}-\mathrm{NMR}\left(\mathrm{CDCl}_{3}, 400 \mathrm{MHz}\right) \delta 0.93(\mathrm{t}, J 7.2 \mathrm{~Hz}, 3 \mathrm{H}$, $\mathrm{OCH}_{2} \mathrm{CH}_{2} \mathrm{CH}_{2} \mathrm{CH}_{3}$ ), 1.40 (sextet, $J 7.2 \mathrm{~Hz}, 2 \mathrm{H}, \mathrm{OCH}_{2} \mathrm{CH}_{2} \mathrm{C}_{2} \mathrm{CH}_{3}$ ), 1.63 (quint, $J$ 7.2Hz, 2H, $\mathrm{OCH}_{2} \mathrm{CH}_{2} \mathrm{CH}_{2} \mathrm{CH}_{3}$ ), 2.71(dd, J 16.8, $\left.11.2 \mathrm{~Hz}, 1 \mathrm{H}, \mathrm{Ha}-4\right), 3.02$ (dd, J 13.6, 7.2 Hz, 1H, Ha-9), 3.11 (dd, $J$ 16.8, 3.2 Hz, 1H, Hb-4), 3.23 (dd, $J$ 13.6, $6.0 \mathrm{~Hz}, 1 \mathrm{H}, \mathrm{Hb}-9), 3.88$ (t, $J$ 7.2Hz, 2H, $\left.\mathrm{OC}_{2} \mathrm{CH}_{2} \mathrm{CH}_{2} \mathrm{CH}_{3}\right), 3.90$ (s, 3H, OCH 3$), 4.66$ (m, 1H, H-3), 6.89 (d, J 8.4 Hz, 1H, ArH), 7.29 $(\mathrm{m}, 5 \mathrm{H}, \mathrm{ArH}), 7.86(\mathrm{~d}, J$ 8.4Hz, $1 \mathrm{H}, \mathrm{ArH}) ;{ }^{13} \mathrm{C}-\mathrm{NMR}\left(\mathrm{CDCl}_{3}, 100 \mathrm{MHz}\right) \delta 13.8,19.1,26.8,32.2$, 41.3, 55.8, 73.0, 78.8, 110.7, 118.0, 126.8, 127.2, 128.6, 129.6, 133.1, 136.2, 143.9, 157.0, 165.4; EIMS (70eV) m/z (rel. intensity, \%) 340 (M+, 22), 250 (8), 249 (52), 194 (11), 193 (100), 166 (9), 165 (34), 91 (6); HRMS Calcd for $\mathrm{C}_{19} \mathrm{H}_{20} \mathrm{O}_{4}: 340.1675$. Found: 340.1677; Anal. Calcd for $\mathrm{C}_{21} \mathrm{H}_{24} \mathrm{O}_{4}$ : C, 74.09; H, 7.11. Found: C, 73.88; H, 7.11.

\section{Acknowledgements}

We are grateful to National Science Council of Taiwan (NSC-99-2113-M-037-007) and Kaohsiung Medical University (N-843) for financial support.

\section{References}

1. Govindachari, T. R.; Patankar, S. J.; Viswanathan, N. Phytochemistry 1971, 10, 1603-1606. http://dx.doi.org/10.1016/0031-9422(71)85032-X

2. Hashimoto, T.; Tori, M.; Asakawa, Y. Phytochemistry 1987, 26, 3323-3330. http://dx.doi.org/10.1016/S0031-9422(00)82497-8

3. Speranza, G.; Manitto, P.; Cassara, P.; Monti, D. Phytochemistry 1993, 33, 175-178. http://dx.doi.org/10.1016/0031-9422(93)85417-P

4. Ito, C.; Mishina, Y.; Litaudon, M.; Cosson, J.-P.; Furukawa, H. Phytochemistry 2000, 53, 1043-1046. 
http://dx.doi.org/10.1016/S0031-9422(99)00442-2

5. Veitch, N. C.; Simmonds, M. S. J.; Blaney, W. M.; Reynolds, T. Phytochemistry 1994, 35, 1163-1166.

http://dx.doi.org/10.1016/S0031-9422(00)94814-3

6. Biswas, K. M.; Mallik, H. Phytochemistry 1986, 25, 1727-1730.

http://dx.doi.org/10.1016/S0031-9422(00)81245-5

7. Magid, A. A.; Voutquenne-Nazabadioko, L.; Moroy, G.; Moretti, C.; Lavaud, C.

Phytochemistry 2007, 68, 2439-2443.

http://dx.doi.org/10.1016/j.phytochem.2007.05.011, PMid:17618658

8. Guimaraes, K. G.; de Souza Filho, J. D.; dos Mares-Guia, T. R.; Braga, F. C.

Phytochemistry 2008, 69, 439-444.

http://dx.doi.org/10.1016/j.phytochem.2007.08.002, PMid:17870137

9. Zhang, X. F.; Xie, L.; Liu, Y.; Xiang, J. F.; Tang, Y. L. J. Mol. Struct. 2008, 891, 87-92. http://dx.doi.org/10.1016/j.molstruc.2008.03.005

10. Kongsaeree, P.; Prabpai, S.; Sriubolmas, N.; Vongvein, C.; Wiyakrutta, S. J. Nat. Prod. 2003, 66, 709-711.

http://dx.doi.org/10.1021/np0205598, PMid:12762815

11. Ghosh, A. K.; Cappiello, J. Tetrahedron Lett. 1998, 39, 8803-8806.

http://dx.doi.org/10.1016/S0040-4039(98)01977-7

12. da Penha, E. T.; Forni, J. A.; Biajoli, A.F. P.; Correia, C. R. D. Tetrahedron Lett. 2011, 52, 6342-6345.

http://dx.doi.org/10.1016/j.tetlet.2011.09.014

13. Mandal, S. K.; Roy, S. C. Tetrahedron 2007, 64, 11050-11057.

http://dx.doi.org/10.1016/j.tet.2008.09.075

14. Ollero, L.; Castedo, L.; Dominguez, D. Synlett 1997, 1047-1048.

http://dx.doi.org/10.1055/s-1997-1538

15. Larock, R.; Yang, H. Synlett 1994, 748-750.

http://dx.doi.org/10.1055/s-1994-22996 
16. (a) Tahara, N.; Fukuda, T.; Iwao, M. Tetrahedron Lett. 2004, 45, 5117-5120.

http://dx.doi.org/10.1016/j.tetlet.2004.04.158

(b) Uchida, K.; Fukuda, T.; Iwao, M. Tetrahedron 2007, 63, 7178-7186.

http://dx.doi.org/10.1016/j.tet.2007.04.092

17. (a) Bonollo, S.; Lanari, D.; Vaccaro, L. Eur. J. Org. Chem. 2011, 14, 2587-2598; and references cited therein.

http://dx.doi.org/10.1002/ejoc.201001693

(b) Schneider, C. Synthesis 2006, 3919-3944; and references cited therein.

http://dx.doi.org/10.1055/s-2006-950348

18. Michelin, R. A.; Mozzon, M.; Bertani, R. Coord. Chem. Rev. 1996, 147, 299-338.

http://dx.doi.org/10.1016/0010-8545(94)01128-1

19. Chen, P. Y.; Chen, H. M.; Chiang, M. Y.; Wang, Y. F.; Li, S. R.; Wang, T. P.; Wang, E. C. Tetrahedron 2012, 68, 3030-3036.

http://dx.doi.org/10.1016/j.tet.2012.02.023

20. (a) Chen, P. Y.; Huang, K. S.; Tsai, C. C.; Wang, T. P.; Wang, E. C. Org. Lett. 2012, 14, 4930-4933.

http://dx.doi.org/10.1021/ol302256y, PMid:22934626

(b) Tsai, J. C.; Li, S. R.; Chiang, M. Y.; Chen, L. Y.; Chen, P. Y.; Lo, Y. F.; Wang, C. H.; Lin, C. N. Wang, E. C. J. Org. Chem. 2009, 74, 8789-8801.

(c) Chen, P. Y.; Wang, T. P.; Chiang, M. Y.; Huang, K. S.; Tzeng, C. C.; Chen, Y. L.; Wang, E.

C. Tetrahedron 2011, 67, 4155-4160.

http://dx.doi.org/10.1016/j.tet.2011.04.070 